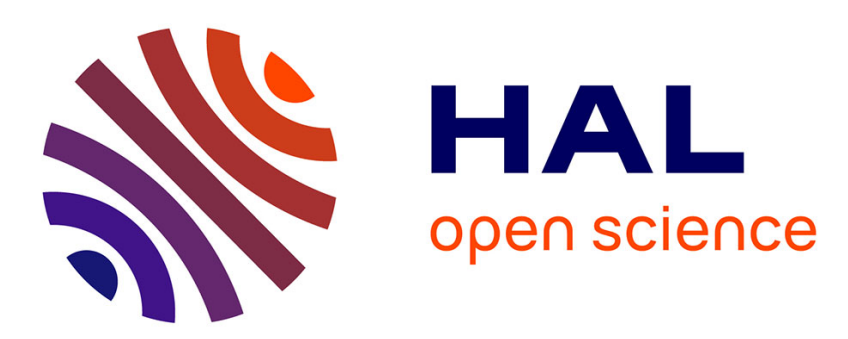

\title{
SIMULATION NUMÉRIQUE DE LA PROPAGATION D'ONDES ACOUSTIQUES DANS LES RÉSEAUX DE TUYAUTERIES
}

\author{
F. Di Costanzo, E. Landel, P. Blanc
}

\section{- To cite this version:}

F. Di Costanzo, E. Landel, P. Blanc. SIMUlATION NUMÉRIQUE DE LA PROPAGATION D'ONDES ACOUSTIQUES DANS LES RÉSEAUX DE TUYAUTERIES. Journal de Physique IV Proceedings, 1992, 02 (C1), pp.C1-479-C1-482. 10.1051/jp4:19921103 . jpa-00251057

\section{HAL Id: jpa-00251057 https://hal.science/jpa-00251057}

Submitted on 1 Jan 1992

HAL is a multi-disciplinary open access archive for the deposit and dissemination of scientific research documents, whether they are published or not. The documents may come from teaching and research institutions in France or abroad, or from public or private research centers.
L'archive ouverte pluridisciplinaire HAL, est destinée au dépôt et à la diffusion de documents scientifiques de niveau recherche, publiés ou non, émanant des établissements d'enseignement et de recherche français ou étrangers, des laboratoires publics ou privés. 


\title{
SIMULATION NUMERIQUE DE LA PROPAGATION D'ONDES ACOUSTIQUES DANS LES RÉSEAUX
} DE TUYAUTERIES

\author{
F. DI COSTANZO, E. LANDEL* et P. BLANC**
}

TECHNICATOME MVI, Parc Club du Golf, BP. 34000, F-13791 Aix en Provence cedex 3, France

*PRINCIPLA $R \&$ D, Zone Portuaire de Brégaillon, BP. 160, F-83503 La Seyne Garibaldi cedex, France

\section{ABSTRACT}

A theoretical study of sound propagation in piping networks has been carried out taking into account complete solutions of the equation of acoustic propagation in fluids.

A computer program has proven to be an efficient and effective tool for the analysis and prediction of sound propagation in ducts. This short paper gives a brief description of the theoretical approach and of the computer program.

\section{INTRODUCTION}

L'étude et la prévision de la propagation des ondes acoustiques dans les circuits hydrauliques ou aérauliques intéressent de nombreux domaines tels que la réduction des bruits induits par les réseaux de canalisation à bord des avions ou à bord des bâtiments de la Marine Nationale.

En particulier, l'amélioration de 1 a discrétion acoustique des réseaux de tuyauteries à bord des sous-marins implique :

- une bonne connaissance de 1 a propagation des ondes acoustiques dans les réseaux de tuyauteries,

- la caractérisation des sources acoustiques,

- la possession d'outils permettant de calculer la réponse acoustique d'un réseau soumis à l'excitation de sources acoustiques (auxiliaires bruyants, singularités, d'écoulement,...) placées en certains éléments du réseau. 
A ce titre, un Logiciel d'Aide à la Conception de TUyauteries Silencieuses (LACTUS) a été développé.

Cet outil informatique permet le calcul de la pression acoustique générée en tout point (noeud) d'un réseau décrit par l'utilisateur du logiciel, les sources acoustiques étant connues.

\section{FORMULATION THEORIQUE}

\subsection{Mise en équation pour une portion du circuit}

On considère un domaine fluide monophasique inclus dans un réseau, limité par des frontières fictives et contenant éventuellement des sources acoustiques.

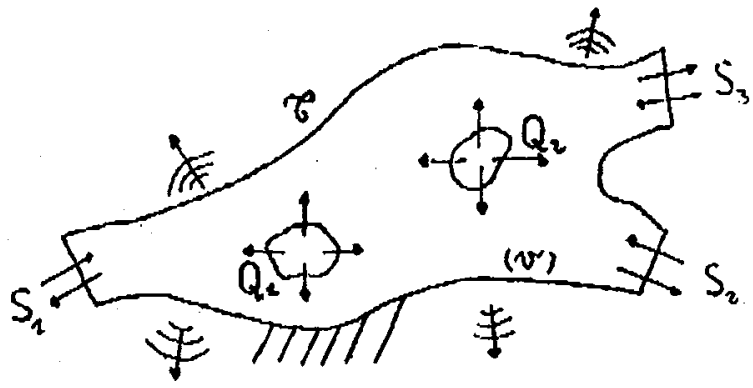

On suppose classiquement que toutes les conditions sont remplies pour pouvoir linéariser les équations et considérer des écoulements irrotationnels. Dans ces conditions, il existe un potentiel des vitesses qui vérifie l'équation d'HELMHOLTZ, lorsque le probTème est harmonique :

$$
\Delta^{2} \Phi+k^{2} \Phi=-\sigma\left(M^{\prime}\right)
$$

$$
(M, t)=\operatorname{Re}\{\Phi(M) \exp (-i \omega t)\}
$$

Dans tout le domaine fluide, le potentiel peut être décrit par l'équation d'HELMHOLTZ-HUYGENS :

$$
\Phi(M)=\iint_{\theta_{v}} \frac{\partial \Phi}{\partial n^{\prime}} G\left(M M^{\prime}\right) d s-\iint_{\partial_{v}} \Phi\left(M^{\prime}\right) \frac{\partial G}{\partial n^{\prime}}\left(M M^{\prime}\right) d s+\iiint_{v} \sigma\left(M^{\prime}\right) G\left(M M^{\prime}\right) d v
$$

La fonction $G$ désigne 1 a fonction de Green du problème dont on suppose qu'elle vérifie l'ensemble des conditions aux limites sur les parois du réseau $C$ :

(4)

$$
i \rho c k \Phi\left(M^{\prime}\right)=Z\left(M^{\prime}\right) \frac{\partial \Phi}{\partial n^{\prime}}\left(M^{\prime}\right)
$$


On suppose, de plus, que le potentiel sur les frontières fictives de la portion de circuit peut se mettre sous la forme suivante, pour laquelle les amplitudes modales sur les frontières $A_{m, n}^{q}$ et $B_{m, n}^{q}$ sont inconnues :

(5)

$$
\Phi\left(M^{\prime}\right)=\sum_{m, n}\left[A_{m, n}{ }^{q} \exp \left(i k_{z} z\right)+B_{m, n}^{q} \exp \left(-i k_{z} z\right)\right] \psi_{m, n}^{q}(r, \theta)
$$

Aux basses fréquences, cette série peut être tronquée, si bien que le problème peut être valablement décrit avec un nombre $\mathrm{Ni}$ fini d'inconnues. En utilisant les propriétés d'orthogonalité des fonctions de forme $\psi^{q} m, n$, on montre que les amplitudes"modales doivent vérifier un ensemble de relations qui peuvent se mettre sous forme matricielle :

$$
[R]\left(\begin{array}{l}
A_{m, n}^{q} \\
B_{m, n}^{q}
\end{array}\right)=\left(S_{m, n}^{q}\right)
$$

où [R] est une matrice $(\mathrm{Ni} / 2, \mathrm{Ni})$

\subsection{Raccordement des éléments}

Un réseau hydraulique est séparé en différents éléments dont les matrices de transfert sont connues. La liaison entre ces éléments pour toutes les frontières fictives, s'obtient en écrivant la continuité du potentiel et de sa dérivée normale.

Les fonctions de forme $\psi^{q_{m}, n}$ étant identiques de part et d'autre de la frontière, il est immédiat que les amplitudes modales sont égales :

$$
\left\{\begin{array}{lll}
A_{m, n} q=A_{m, n}^{\prime} & \forall m \forall n \\
B_{m, n}^{q}=B_{m, n}^{\prime} & \forall m \forall n
\end{array}\right.
$$

\section{DESCRIPTION DU LOGICIEL}

Pour des raisons pratiques, on a limité l'application numérique de cette théorie aux seules ondes planes tout en se réservant la possibilité de traiter $d^{\prime}$ autres types $d^{\prime}$ ondes.

\subsection{Aspects topologiques}

On suppose que le réseau de tuyauteries est constitué $d^{\prime} u n$ ensemble de composants (vannes, clapets, tubes droits, dérivations...) qui se comportent comme des sources acoustiques ou comme des guides d'ondes et peuvent parfois cumuler les deux fonctions.

A ces composants, il convient d'ajouter des composants de type "terminaison" ou "extrémité" (terminaison anéchoïque, terminaison à la mer,...), ce qui revient à imposer des conditions aux limites (section terminale).

Les sources sont introduites sous la forme de discontinuités de la pression ou de la vitesse acoustique au sein des éléments. 


\subsection{Environnement et traitements}

LACTUS est un code modulaire, facilement transportable sur toute machine possédant le FORTRAN 77 et le langage $c$ et ouvert à de multiples développements.

I1 est déjà couplé au logiciel FLOWMASTER de modélisation de réseaux de tuyauteries et $d^{\prime}$ analyse des régimes permanents associés aux écoulements de fluides, et peut s'adapter à tout autre système, qu'il s'agisse d'un logiciel de calcul ou de post-traitement...

Le logiciel LACTUS calcule, dans un réseau décrit par l'utilisateur, un grand nombre de variables telles que :

- 1a pression acoustique en chaque noeud du réseau,

- la pression acoustique rayonnée en mer ramenée à 1 mètre,

- le niveau d'intensité acoustique dans les salles.

\section{CONCLUSION}

La présente étude nous a conduit à réaliser un logiciel de calcul d'un emploi facile, spécifiquement conçu pour traiter les problèmes de propagation d'ondes planes dans les réseaux de tuyauteries. Il offre, en particulier, les possibilités suivantes :

- étudier et visualiser la réponse acoustique d'un circuit complexe composé de singularités passives diverses (élargissement brusque, té,...), de sources actives (pompes, compresseurs, ...) avec recherche des résonances acoustiques,

- optimiser 1'implantation du (ou des) silencieux qui sera monté dans un circuit quelconque compte tenu de la position du spectre et du type (pression ou vitesse) de la (ou des) source acoustique,

- etc.

De plus, nous avons pu tester la validité de nos calculs sur différents cas connus expérimentalement ou analytiquement.

Des développements ultérieurs sont dores et déjà envisagés. Citons en particulier, la possibilité de traiter des modes de propagation d'ordre radial supérieur à 1 .

\section{REMERCIEMENTS}

Nous tenons à remercier vivement les responsables de la DCN/CHERBOURG pour le soutien qu'ils ont apporté à ces travaux. 